\title{
Disintegration locations in ${ }^{7} \mathrm{Li} \rightarrow{ }^{8} \mathrm{Be}$ transfer-triggered breakup at near-barrier energies
}

\author{
E. C. Simpson, K. J. Cook, D. H. Luong, Sunil Kalkal, I. P. Carter, M. Dasgupta, D. J. Hinde, and E. Williams \\ Department of Nuclear Physics, Research School of Physics and Engineering, Australian National University, ACT 2601, Australia
}

(Received 20 October 2015; published 3 February 2016)

\begin{abstract}
Background: At above-barrier energies, complete fusion cross sections in collisions of light weakly bound nuclei with heavy target nuclei are suppressed when compared to well-bound nuclei. Breakup of the projectilelike nucleus was proposed to be the cause. In addition to direct breakup, breakup following transfer was shown to be substantial.

Purpose: We investigate breakup in reactions with ${ }^{7} \mathrm{Li}$, triggered by sub-barrier proton pickup to unbound states in ${ }^{8} \mathrm{Be}$, which subsequently separate into two $\alpha$ particles.

Method: Measurements of sub-barrier disintegration of ${ }^{7} \mathrm{Li}$ on a ${ }^{58} \mathrm{Ni}$ target were made using the Heavy Ion Accelerator Facility at the Australian National University. Combining the experimental results with classical simulations of post-breakup acceleration, we study the sensitivity of $\alpha-\alpha$ energy and angle correlations to the proximity of disintegration to the target (proton donor) nucleus.

Results: The simulations indicate that disintegration as the colliding nuclei approach each other leads to large angular separations $\theta_{12}$ of the $\alpha$ fragments. The detectors allow for a maximum opening angle of $\theta_{12}=132^{\circ}$, such that the present experiment is largely insensitive to breakup occurring when the collision partners approach each other. The data are consistent with disintegration of (a) the $0^{+8} \mathrm{Be}$ ground state far from the targetlike nucleus, and (b) the $2^{+8} \mathrm{Be}$ resonance near the targetlike nucleus when the ${ }^{8} \mathrm{Be}$ is receding from the targetlike nucleus.

Conclusions: The present results shed light on the near-target component of transfer-induced breakup reactions. The distribution of events with respect to the opening angle of the $\alpha$ particles, and the orientation of their relative velocity with respect to the velocity of their center of mass, gives insights into their proximity to the target at the moment of breakup. Further measurements with larger angular coverage and more complete simulations are required to fully understand the influence of breakup on fusion.
\end{abstract}

DOI: 10.1103/PhysRevC.93.024605

\section{INTRODUCTION}

Complete fusion of weakly bound nuclei such as ${ }^{6,7} \mathrm{Li}$ and ${ }^{9} \mathrm{Be}$ in reactions with heavy target nuclei is known to be significantly suppressed [1-6] at above-barrier energies with respect to well-bound nuclei and single-barrier penetration model calculations. The effect was found in a large number of systems, with the typical suppression of the complete fusion cross section around $\sim 30 \%$. It was suggested [1] that the cause is breakup of the projectile into fragments before passing inside the fusion barrier, reducing the likelihood that the complete projectile will fuse with the target.

The weak-binding and ground-state cluster structure of these projectiles makes them susceptible to breakup through direct population of their continuum states: ${ }^{6} \mathrm{Li}$ into $\alpha+d$, ${ }^{7} \mathrm{Li}$ into $\alpha+t$, and ${ }^{9} \mathrm{Be}$ into $2 \alpha+n$. However, because neighboring nuclei are particle unbound, transfer reactions may lead to ejectiles that disintegrate into two or more fragments [7-9]. For example, proton pickup by ${ }^{7} \mathrm{Li}$ forms unbound ${ }^{8} \mathrm{Be}$, yielding two $\alpha$ particles. One- and two-neutron transfer, populating states above the $\alpha+d$ threshold in ${ }^{6} \mathrm{Li}$ and in $\alpha+p$ unbound ${ }^{5} \mathrm{Li}$, respectively, can also trigger breakup. These modes have been identified in experiments measuring coincidences of two breakup fragments, both at energies above [9,10] and below [7,11-13] the barrier energy. For ${ }^{6} \mathrm{Li},{ }^{7} \mathrm{Li}$, and ${ }^{9} \mathrm{Be}$, transfer-induced breakup is as important as direct breakup with heavy ${ }^{144} \mathrm{Sm},{ }^{207,208} \mathrm{~Pb}$, and ${ }^{209} \mathrm{Bi}$ target nuclei $[12,13]$. Thus in this work we define breakup as any process which leads to the disintegration of the projectilelike nucleus into two fragments, either directly or following nucleon transfer.

The nuclear structure of the unbound state populated prior to breakup influences the subsequent breakup process. Thus there are significant variations between different projectiles and reaction channels. For example, narrow resonances have been identified in $\alpha+d$ and $\alpha+\alpha$ breakup channels, associated with states in ${ }^{6} \mathrm{Li}$ and ${ }^{8} \mathrm{Be}$ [12-15]. These states have lifetimes $\left(>10^{-20} \mathrm{~s}\right)$ that are much longer than the collision times scales $\left(\sim 10^{-21} \mathrm{~s}\right)$, and so will not be able to suppress fusion. Other broad resonances are known (e.g., in ${ }^{5} \mathrm{Li}$ and ${ }^{8} \mathrm{Be}$ ), which may disintegrate into fragments prior to reaching the fusion barrier, and provide a mechanism by which fusion can be suppressed.

Thus the location of breakup relative to the targetlike nucleus is critical to fusion suppression: Only breakup prior to the projectilelike nucleus being absorbed inside the fusion barrier can suppress fusion. However, the characterization of the breakup process itself is difficult at energies above the barrier. Here, absorption of the fragments following breakup is significant, limiting coincidence measurements of the fragments and complete kinematical reconstruction of the event. In contrast, detailed information on breakup mechanisms can be obtained unambiguously at sub-barrier energies, where capture of the fragments is minimal. At sub-barrier energies breakup prior to the fusion barrier, as illustrated in Fig. 1(a), translates into breakup before the turning point $R_{0}$ (the incoming trajectory). Breakup after this point, when the reactants are receding from each other 

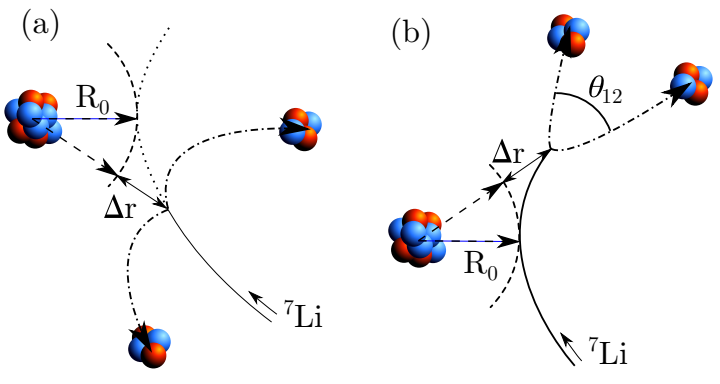

FIG. 1. ${ }^{7} \mathrm{Li}$ projectile trajectories (solid and dotted lines) corresponding to a distance of closest approach $R_{0}$ (dashed arcs). Proton pickup occurs at some point on the solid trajectory to form ${ }^{8} \mathrm{Be}$. Disintegration of ${ }^{8} \mathrm{Be}$ into two $\alpha$ particles can occur either (a) approaching (incoming trajectory) or (b) receding from (outgoing trajectory) the targetlike nucleus. The radial distance relative to $R_{0}$ at which breakup occurs is denoted by $\Delta r$. The asymptotic opening angle of the two fragments, following post-acceleration by the target, is $\theta_{12}$. Events resulting in large angular separations $\theta_{12}$ correspond to large relative energies of the $\alpha$ fragments.

(the outgoing trajectory) is not relevant to fusion suppression, because there is no outgoing trajectory in fusion.

By studying the detail of sub-barrier breakup, we can then infer the influence of breakup on above-barrier fusion using models (e.g., [16]). This work concentrates on the first step, specifically in obtaining information on (a) whether sub-barrier breakup occurs when the projectile- and targetlike nuclei are approaching or receding from each other, and (b) the internuclear distance at the point of breakup. Both are critical to fusion suppression. We consider the case of proton pickup by ${ }^{7} \mathrm{Li}$ from a ${ }^{58} \mathrm{Ni}$ target at sub-barrier energies, producing two $\alpha$ particles - a channel found to be strong in reactions with heavy target nuclei in the lead region $[12,13]$. Using classical dynamical simulations we examine the effect of different locations of near-target breakup on the energy and angular correlations of the two $\alpha$ fragments produced in the reaction.

\section{NEAR-TARGET AND ASYMPTOTIC BREAKUP}

\section{A. Experiment}

The experiment presented here was performed using a beam of $13.1 \mathrm{MeV}{ }^{7} \mathrm{Li}$ nuclei provided by the 14UD tandem electrostatic accelerator at the Australian National University Heavy Ion Accelerator Facility. This beam energy is approximately $96 \%$ of the ${ }^{7} \mathrm{Li}-{ }^{58} \mathrm{Ni}$ fusion barrier $V_{B}$, where $V_{B}$ was estimated using the Sao Paulo potential [17]. Charged particles were detected with the Breakup Array for Light Nuclei (BALiN [18]), a large area double-sided silicon strip detector array. In the present experiment this was placed at backward angles, covering $\theta \approx 114^{\circ}-165^{\circ}$ with respect to the beam axis, and up to $210^{\circ}$ in $\phi$. The states of ${ }^{57} \mathrm{Co}$ populated are consistent with those strongly populated in ${ }^{58} \mathrm{Ni}(t, \alpha){ }^{57} \mathrm{Co}$ and ${ }^{58} \mathrm{Ni}\left(d,{ }^{3} \mathrm{He}\right){ }^{57} \mathrm{Co}$ proton-pickup reactions [19-21]. The data presented here are for the ${ }^{57} \mathrm{Co} 2.9811 / 2^{+}$state, which is near the optimal $Q$ value for population of excited states in ${ }^{8} \mathrm{Be}$ near $3 \mathrm{MeV}$. The experimental setup and analysis are

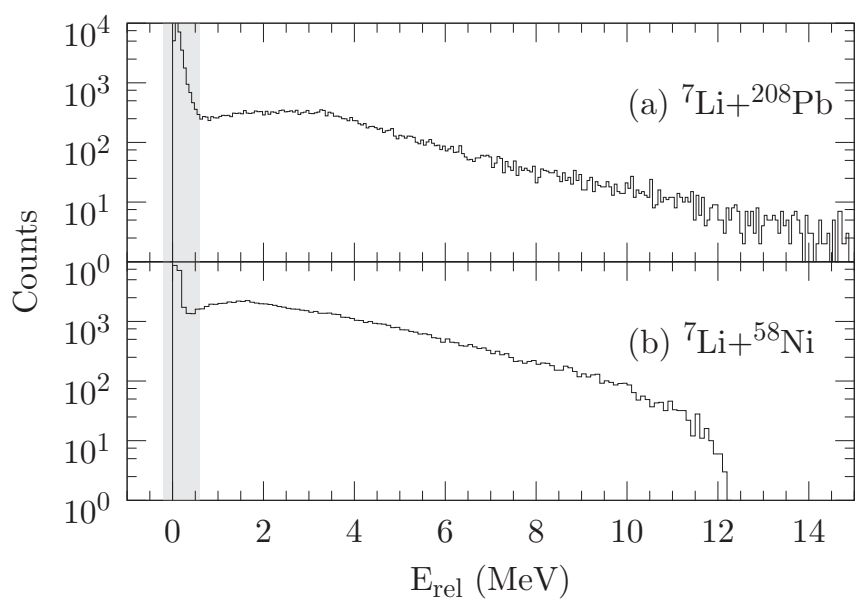

FIG. 2. Relative energy distribution for the two $\alpha$ fragments following proton-pickup-induced disintegration of ${ }^{7} \mathrm{Li}$ interacting with (a) a ${ }^{208} \mathrm{~Pb}$ target at $29 \mathrm{MeV}$ and (b) a ${ }^{58} \mathrm{Ni}$ target at $13.1 \mathrm{MeV}$. At lower energies a prominent peak associated with the ${ }^{8} \mathrm{Be}$ ground state is seen highlighted by the shaded region. In both cases a second bump is seen at larger $E_{\text {rel }}$, before the distribution decays exponentially.

essentially identical to earlier experiments using a ${ }^{9} \mathrm{Be}$ beam, with a complete description given in Ref. [11]. Here we focus on the interpretation of the results.

\section{B. Fragment relative energies}

Previous work [11-13] had characterized breakup using the measured relative energy distributions of the resulting $\alpha$ particles, $E_{\text {rel }}=\frac{1}{2} \mu v_{12}^{2}$, where $v_{12}$ is the relative velocity of the two fragments and $\mu$ their reduced mass. With fragments of masses $m_{1}$ and $m_{2}$, the relative energy can be deduced from their individual energies $E_{1}$ and $E_{2}$, and the opening angle of their velocity vectors $\theta_{12}$ :

$$
E_{\mathrm{rel}}=\frac{m_{2} E_{1}+m_{1} E_{2}-2 \sqrt{m_{1} E_{1} m_{2} E_{2}} \cos \theta_{12}}{m_{1}+m_{2}} .
$$

The measured relative energy distributions for the two $\alpha$ particles resulting from ${ }^{7} \mathrm{Li}$ proton pickup with the ${ }^{58} \mathrm{Ni}$ target is shown in the bottom panel of Fig. 2. This is compared to the distribution produced by the same proton-pickup reaction of ${ }^{7} \mathrm{Li}$ on a ${ }^{208} \mathrm{~Pb}$ target [13], shown in the top panel. In both cases the collision energy is below that of the respective fusion barriers. The maximum relative energy with the ${ }^{58} \mathrm{Ni}$ target is limited by the maximum fragment opening angle $\theta_{12}$ allowed by the detector system. Data from both ${ }^{208} \mathrm{~Pb}$ and ${ }^{58} \mathrm{Ni}$ targets show a peak at low $E_{\text {rel }}$ (indicated by the gray band) on a broad, largely featureless background which decays exponentially as $E_{\text {rel }}$ increases.

At the point of breakup, the relative energy of the $\alpha$ particles reflects the intrinsic excitation distribution of ${ }^{8} \mathrm{Be}$ resulting from the transfer. This in turn depends on the energy, shape, and width of resonances, in addition to effects arising from the production mechanism (e.g., the favored $Q$-value window) [22-24]. If the fragments only interacted weakly with the targetlike nucleus following breakup, for example, in ${ }^{7} \mathrm{Li}(d, n){ }^{8} \mathrm{Be}$ or ${ }^{9} \mathrm{Be}(p, d){ }^{8} \mathrm{Be}$, the final measured relative 
energy distribution would reflect this intrinsic distribution. However, for both ${ }^{208} \mathrm{~Pb}$ and ${ }^{58} \mathrm{Ni}$ targets there are potentially strong Coulomb interactions of the $\alpha$ particles with the targetlike nucleus following breakup, which change their trajectories, distorting the relative energy distribution. The degree to which this occurs depends on whether the breakup occurs asymptotically far from the target or near the target.

\section{Asymptotic breakup}

The ${ }^{8} \mathrm{Be}$ ground state is an extreme example of asymptotic breakup. At just $0.092 \mathrm{MeV}$ above the $\alpha \alpha$ threshold, it has a width $\Gamma=5.6 \mathrm{eV}$ corresponding to a lifetime of $1.17 \times 10^{-16} \mathrm{~s}[25]$. As such, it separates into two $\alpha$ particles very far from the target nucleus (asymptotic breakup). There is, therefore, essentially no acceleration of the separated $\alpha$ particles from the Coulomb field of the target, and the relative energy observed remains characteristic of the excitation of the state (broadened by instrumental resolution, see Ref. [13]). A well-defined peak is then clearly visible in the $E_{\text {rel }}$ distribution, as found for both ${ }^{58} \mathrm{Ni}$ and ${ }^{208} \mathrm{~Pb}$ targets and indicated by the shaded region in Fig. 2. The asymptotic breakup component is unable to influence fusion at above-barrier energies, as breakup will not occur before the projectilelike nucleus has reached the fusion barrier.

\section{Near-target breakup}

Disintegration of the projectilelike nucleus - in this case, ${ }^{8} \mathrm{Be}$-into fragments close to the target is able to suppress fusion, and was previously referred to as prompt breakup. Following breakup the $\alpha$ particles are accelerated by their Coulomb interactions with the target, resulting in a wide range of relative energies and the smoothly varying background shown in Fig. 2. Because half of the proton transfer should occur on the incoming trajectory and half on the outgoing trajectory, it was previously assumed $[12,13]$ that the half of near-target breakup (from the incoming trajectory) would contribute to complete fusion suppression. However, a more detailed understanding is desirable.

It was noted [11] that near-target breakup events may proceed via the ${ }^{8} \mathrm{Be} 3.122-\mathrm{MeV}^{+}$state. Because of its large width $\Gamma=1.513 \mathrm{MeV}$ [25] the decay was treated as effectively instantaneous. Although the initial relative energy of the fragments should be reasonably well defined by the resonance energy, the strong Coulomb field of the target causes them to accelerate rapidly. This acceleration causes their relative energy to increase or decrease depending on their initial orientation with respect to the target. The magnitude of the acceleration, determined by both the charge of the target and distance to the target at the point of breakup, determines how strong the distortions of the relative energy distribution are from the intrinsic resonance line shape.

Because of this acceleration it is not immediately clear how the final $E_{\text {rel }}$ distribution is sensitive to the $2^{+}$state and its properties. The final relative energy distributions for both ${ }^{58} \mathrm{Ni}$ and ${ }^{208} \mathrm{~Pb}$ targets exhibit a broad, flat maximum in the region $E_{\mathrm{rel}} \approx 1-3 \mathrm{MeV}$ (see Fig. 2). This is, however, target dependent: The ${ }^{58} \mathrm{Ni}$ case shows a more well-defined peak at lower energy than the flat maximum found for ${ }^{208} \mathrm{~Pb}$. Given the large difference in charge between the two targets, that the two are different is not surprising. As the post-breakup acceleration with the ${ }^{58} \mathrm{Ni}$ target is considerably weaker, the resulting relative energy distribution is more likely to retain features of the $2^{+}$resonance. We thus focus on the ${ }^{58} \mathrm{Ni}$ target in this work, in an effort to understand the prompt breakup and post-breakup acceleration in more detail.

\section{CHARACTERIZING NEAR-TARGET BREAKUP}

To better characterize near-target breakup we consider two further quantities - the difference of fragment energies $\left|E_{1}-E_{2}\right|$, and the correlation between the opening angle of the fragments $\theta_{12}$ and the orientation of their relative velocity with respect to the velocity of their center of mass. Both quantities have a well-defined distribution when breakup occurs far from the target. We consider these asymptotic limits, and the deviations from them observed in the experimental data, in the following.

\section{A. Fragment energy differences}

Where breakup results in two fragments of the same mass, the maximum difference of energies occurs when their relative velocity is aligned with the velocity of their center of mass, such that $\theta_{12}=0^{\circ}$ or $180^{\circ}$. This quantity is given by

$$
\left|E_{1}-E_{2}\right| \leqslant 2 \sqrt{E_{\mathrm{rel}}\left(E_{0}-E_{\mathrm{rel}}\right)},
$$

where $E_{0}$ is the total energy $E_{0}=E_{1}+E_{2}$. If the breakup occurs asymptotically far from the target, the distribution of $\left|E_{1}-E_{2}\right|$ should run from zero to this $E_{\text {rel-dependent }}$ maximum. Furthermore, if the decay is isotropic we should find that the intensity of events is independent of $\left|E_{1}-E_{2}\right|$ for a given relative energy [8].

The measured distribution of $\left|E_{1}-E_{2}\right|$ with $E_{\text {rel }}$ is shown in Fig. 3. The curved dashed line shows the maximum

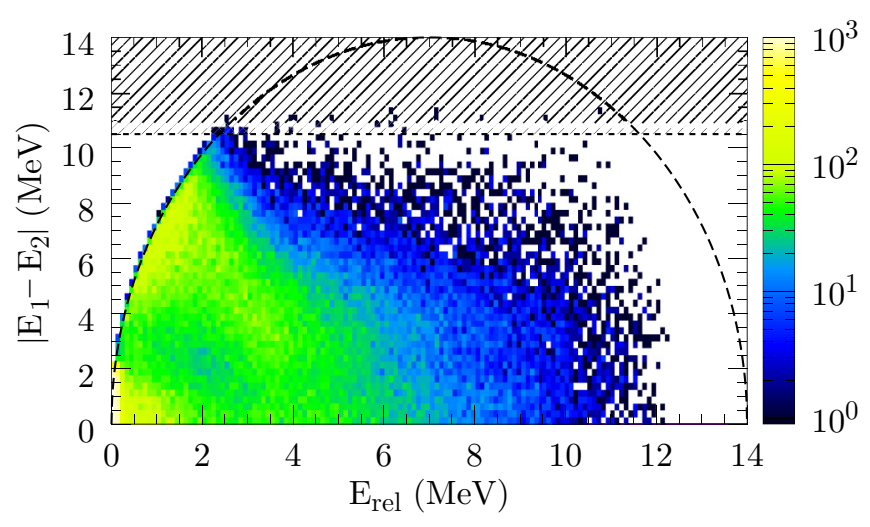

FIG. 3. The distribution of coincidence events according to their relative energy $E_{\text {rel }}$ and difference of energies of the fragments $\mid E_{1}-$ $E_{2} \mid$. The dashed arc shows the expected maximum difference, which should be attained for all $E_{\text {rel }}$ in the absence of the target nucleus. The hatched area shows the detection limit assuming a threshold $\alpha$ energy of $1.75 \mathrm{MeV}$. Both estimates assume a constant total energy $E_{1}+E_{2}=14 \mathrm{MeV}$. 
allowed energy difference $\left|E_{1}-E_{2}\right|$ as a function of $E_{\text {rel }}$, assuming a total energy $E_{1}+E_{2}=14 \mathrm{MeV}$. The hatched area shows the detection limit based on the minimum detectable $\alpha$-particle energy from energy losses and electronics thresholds.

The ground state of ${ }^{8} \mathrm{Be}$ appears as an intense band at low relative energy in the bottom left corner of the plot $E_{\text {rel }} \lesssim 0.2$ $\mathrm{MeV}$, and reaches the limiting $\left|E_{1}-E_{2}\right|$ value. As expected, the intensity is independent of $\left|E_{1}-E_{2}\right|$ for this asymptotic, isotropic decay. For slightly higher relative energies with $0.2 \lesssim E_{\text {rel }} \lesssim 2.4 \mathrm{MeV}$ the events continue to reach to the limiting value, although the intensity is no longer constant with $\left|E_{1}-E_{2}\right|$ for a given $E_{\text {rel }}$. Above $E_{\text {rel }} \approx 2.4 \mathrm{MeV}$ the intensity becomes increasingly focused towards $\left|E_{1}-E_{2}\right|=0$. These events are likely to be from breakup near the target, where the majority of energy is stored in the fragment-target potentials. This energy is then released in the (largely) independent interactions of the fragments with the target, resulting in fragments with similar energies. Aside from those events clearly associated with the ${ }^{8} \mathrm{Be}$ ground state, almost all breakup occurs sufficiently close to the targetlike nucleus such that the post-disintegration acceleration by the targetlike nucleus alters the fragment trajectories.

\section{B. Fragment angular correlations}

To further probe breakup close to the targetlike nucleus, it is useful to first consider the expected fragment angular correlations for a given relative energy for asymptotic breakup. In particular, we consider how the orientation of the relative velocity of the two fragments is correlated with their opening angle $\theta_{12}$. For the limit of asymptotic breakup, the relevant velocity diagram is shown in Fig. 4. The laboratory velocities of the fragments are $v_{1}$ and $v_{2}$, deduced from the measured energies $E_{1}$ and $E_{2}$. The velocity of their center of mass is $v_{c}$. The velocities of the fragments in the ${ }^{8} \mathrm{Be}$ rest frame are $u_{1}$ and $u_{2}$, deduced from the relative energy $E_{\text {rel }}=\frac{1}{2} \mu\left(u_{1}+u_{2}\right)^{2}$ and conservation of momentum, where $\mu$ is the reduced mass of the fragment pair. The angle $\beta$ is the orientation of the relative velocity with respect to the velocity of the center of mass of the fragments.

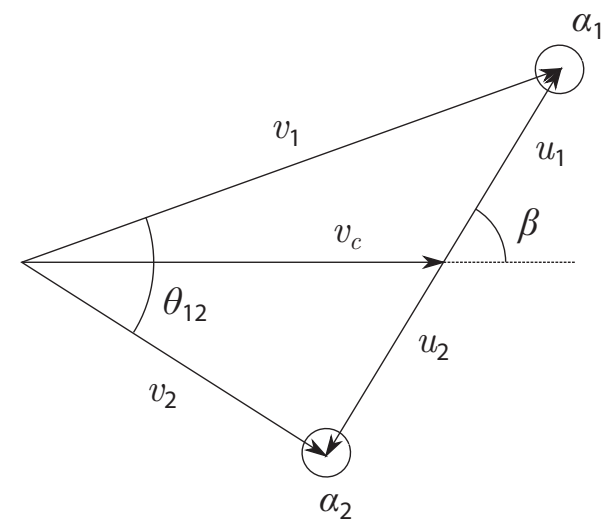

FIG. 4. Notation for fragment velocities for isolated disintegration. The laboratory frame opening angle is $\theta_{12}$. The orientation of the relative velocity with respect to that of the center of mass is $\beta$.

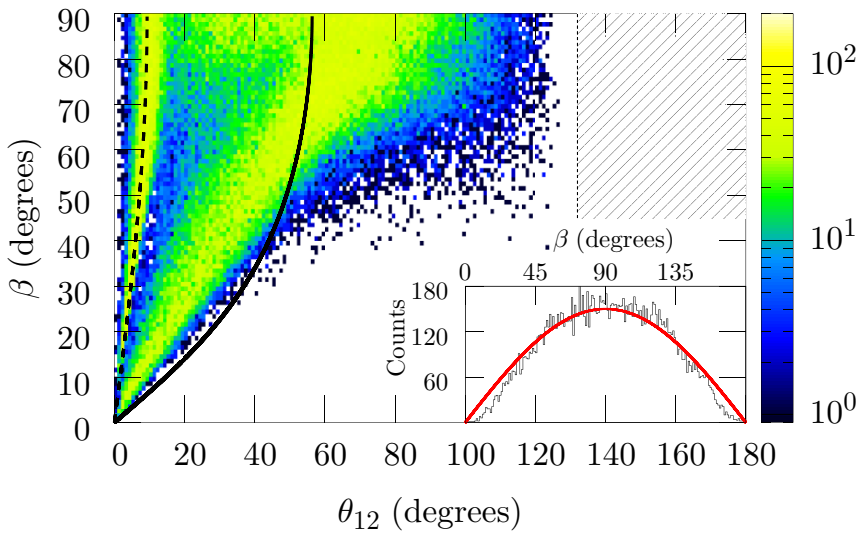

FIG. 5. Plot of $\beta$ against $\theta_{12}$ showing the present measurements. The lines indicate the expected correlation for asymptotic breakup for an initial relative energy of $0.092 \mathrm{MeV}$ (dashed) and $3.122 \mathrm{MeV}$ (solid). The hatched area shows the expected $\theta_{12}$ inaccessible with the current detector configuration. In reality, the efficiency will decrease as this region is approached. The inset shows the projection of the $0.092-\mathrm{keV}$ band onto $\beta$, with the red line showing the expected $\sin \beta$ isotropic distribution. See text for further discussion.

For a fixed initial excitation energy $E_{x}$ (equivalent to $E_{\text {rel }}$ in the asymptotic case), there is a direct mapping between $\theta_{12}$ and $\beta$. This angle can be deduced directly from the measured opening angle $\theta_{12}$, the laboratory energies of the fragments $E_{1}$ and $E_{2}$ and their relative energy $E_{\text {rel }}$. This angle $\beta$ is given by

$$
\sin \beta=\frac{v_{1} v_{2} \sin \theta_{12}}{\left(v_{2}^{2} u_{1}^{2}+v_{1}^{2} u_{2}^{2}+2 u_{1} u_{2} v_{1} v_{2} \cos \theta_{12}\right)^{1 / 2}} .
$$

In symmetric disintegration such as ${ }^{8} \mathrm{Be} \rightarrow 2 \alpha$, the maximum opening angle $\theta_{12}$ will come when $\beta=90^{\circ}$ and $E_{1}=E_{2}$. The smallest $\theta_{12}$ are generated when the relative motion of the $\alpha$ particles is aligned with the direction of motion of their center of mass with $\beta=0^{\circ}$ or $180^{\circ}$, and the difference of energies $\left|E_{1}-E_{2}\right|$ is then maximized.

The experimentally observed correlation between $\beta$ and $\theta_{12}$ is shown in Fig. 5. Because both fragments have the same mass the figure is symmetric about $\beta=90^{\circ}$, and we have folded the data about this value. In the present detector configuration, the detectors cover angles forward to $\theta \approx 114^{\circ}$ with up to $210^{\circ}$ coverage in $\phi$. This allows detection of pairs of fragments with a maximum possible opening angle of $\theta_{12} \approx 132^{\circ}$ although the efficiency will be small for the largest values.

The data show two bands. At small $\theta_{12}$ a band of events can be seen resulting from decay of the ground state of ${ }^{8} \mathrm{Be}$. The small relative energy of the $\alpha$ particles gives angular separations up to $\sim 10^{\circ}$ and follows the expected asymptotic disintegration curve from Eq. (3) (dashed curve). The width of the band is primarily from the angular resolution in the experiment-the physical width of the ground state is negligible. Projected onto $\beta$ (shown in the inset), these events show a $\sin \beta$ intensity pattern, consistent with isotropic emission. The reduced intensity at the extremes of $\beta$ result from small angular separations $\theta_{12}$, where both $\alpha$ particles arrive in the same pixel. These events cannot be registered as a coincidence event in the detector array. 
A second band of events is also apparent, extending to larger $\theta_{12}$. That such a band emerges from the relatively featureless $E_{\text {rel }}$ distribution [nonshaded region of Fig. 2(b)] is significant. The solid line shows the result expected from asymptotic disintegration for an initial excitation energy equal to the ${ }^{8} \mathrm{Be}$ $2^{+}$energy. This limit provides a useful benchmark: Although clearly the band is not asymptotic, the data cover a similar range of $\theta_{12}$, suggesting that the prompt breakup does indeed proceed via the ${ }^{8} \mathrm{Be} 2^{+}$resonance. As the breakup position moves inwards towards the distance of closest approach $R_{0}$, we would expect increasingly strong deviations from the asymptotic limit. If breakup occurs close to the target, the potential gradient is large and the fragments will accelerate rapidly away from the targetlike nucleus, strongly eroding the asymptotic $\beta$ vs $\theta_{12}$ correlation (solid line in Fig. 5).

How the post-breakup acceleration changes these correlations will depend on (a) the orientation of the $\alpha$ particles with respect to the target, and (b) how close the fragments are to the target. If the $\alpha$ particles are aligned perpendicular to the target field, the post-acceleration will tend to increase their relative energy and increase $\theta_{12}$. Conversely, if aligned parallel to the target field the stronger acceleration for the $\alpha$ nearest the target will tend to reduce $E_{\mathrm{rel}}$ and reduce $\theta_{12}$. Qualitatively, this seems consistent with the deviations from asymptotic breakup shown in Fig. 5. Thus the correlation of the observables presented here appears to provide insights into the location of breakup.

\section{MODELING POST-BREAKUP ACCELERATION}

To investigate further we need to understand how the postbreakup acceleration of the $\alpha$ particles by the target alters the correlation of $\beta$ and $\theta_{12}$, and understand how the changes are sensitive to the proximity to the target at the point of breakup. To this end, we make simulations using a modified version of the three-dimensional classical dynamical model PLATYPUS $[16,26]$. The simulations model breakup using a ${ }^{8} \mathrm{Be}$ pseudoprojectile, with the incident energy of the ${ }^{8} \mathrm{Be}$ chosen to match the distance of closest approach attained by the physical ${ }^{7} \mathrm{Li}$ projectile incident on the ${ }^{58} \mathrm{Ni}$. The classical trajectory of the ${ }^{8} \mathrm{Be}$ and ${ }^{57} \mathrm{Co}$ target are tracked, and at some point the ${ }^{8} \mathrm{Be}$ disintegrates into two $\alpha$ particles. The interactions between the $\alpha$ particles and target are then followed until their separations are large.

Here our interest lies in how the $\theta_{12}-\beta$ correlation changes with the proximity of breakup to the targetlike nucleus. We thus make simulations using a fixed point of disintegration at radial distance $r=R_{0}+\Delta r$ on the projectile-target trajectory, either before or after the distance of closest approach (see Fig. 1). A small effective $Q$ value is added to the relative motion of the ${ }^{8} \mathrm{Be}$ and target to attain the correct total fragment energy $E_{1}+E_{2}$ found in experiment (see the Appendix).

On the premise that near-target breakup originates from the ${ }^{8} \mathrm{Be} 2^{+}$resonance, we assume an initial relative energy for the $\alpha$ particles appropriate for this state $\left(E_{x} \equiv E_{R}=3.122 \mathrm{MeV}\right)$. At the moment of breakup, the $\alpha$ particles are placed with separation $\vec{d}_{12}$ equal to their mutual barrier radius, with the orientation of $\vec{d}_{12}$ assumed to be isotropic. The $\alpha$ particles then move radially away from each other, and are accelerated by their mutual potential and their interactions with the target.
For each set of calculations 500 events are simulated. The only factor varying is the orientation of the initial separation of the fragments $\vec{d}_{12}$. To gauge the sensitivity to $E_{x}$ we make calculations covering the width of the resonance $\left(E_{x}=\right.$ $E_{R} \pm \Gamma / 2$ ). The calculations are made for fixed projectiletarget relative angular momentum-typically $L=0 \hbar$, although illustrative calculations with $L=4 \hbar$ and $8 \hbar$ are also shown.

The breakup distances $\left(R_{0}+\Delta r\right)$ given are the distance at which disintegration into two $\alpha$ particles occurs and they move apart, rather than where the transfer reaction itself happens. This definition is used because some finite time will be required to tunnel or pass over the $\alpha-\alpha$ barrier. If the $\alpha$ particles remain spatially localized (i.e., inside their mutual barrier) until the fusion barrier with the target is reached, fusion will not be suppressed. Therefore this definition of the breakup point is most relevant in this work. This definition differs from the original implementation of the PLATYPUS model, where the initial separation approximated the size of the ${ }^{8} \mathrm{Be}$ ground state [16]. It is consistent with the definition of breakup used in earlier classical Monte Carlo models [3,27].

\section{A. Turning point breakup}

We first consider breakup of the ${ }^{8} \mathrm{Be}$ near the distance of closest approach of the projectile and target. Based on the measured energy dependence of the ${ }^{7} \mathrm{Li}$ proton pickup probability, one can fit an exponentially decaying transfer probability function [28,29]. This is an integral quantity-it accounts for the total transfer probability for the whole trajectory, and is written in terms of the distance of closest approach $R_{0}$ :

$$
P\left(R_{0}\right) \propto \exp \left(-\gamma R_{0}\right) .
$$

Although not used here where the calculations use a fixed breakup position, the exponential dependence means that the transfer probability should be strongly peaked about the distance of closest approach. A local breakup probability is used within the standard PLATYPUS code to determine the distribution of points where breakup is assumed to occur [16]. It is assumed to have the same exponential slope as the integrated quantity given above. In the present case the slope of the exponential was determined from the energy dependence of the measured transfer probability to be $\gamma \approx 1.02 \mathrm{fm}^{-1}$. Assuming that the local probability per unit time step along the trajectory has the same exponential form as the integral probability, and assuming a sub-barrier Coulomb trajectory, we estimate that $84 \%$ of reactions should occur within $1 \mathrm{fm}$ radial distance of $R_{0}$.

The strong peaking about $R_{0}$ leads us to first consider simulations of the $\alpha$ fragment correlations for breakup near the distance of closest approach, for $\Delta r= \pm 0.1$ and \pm 1.0 $\mathrm{fm}$. Here the sign of $\Delta r$ is used to indicate whether the point in question is before $(-)$ or after $(+)$ the turning point $R_{0}$ (see Fig. 1). The results of the calculations are compared to the experimental data in Fig. 6(a). For each fixed breakup distance, the only factor varying is the orientation of the initial relative velocity of the fragments. Because in these calculations the projectile-target angular momentum is zero, there is axial 


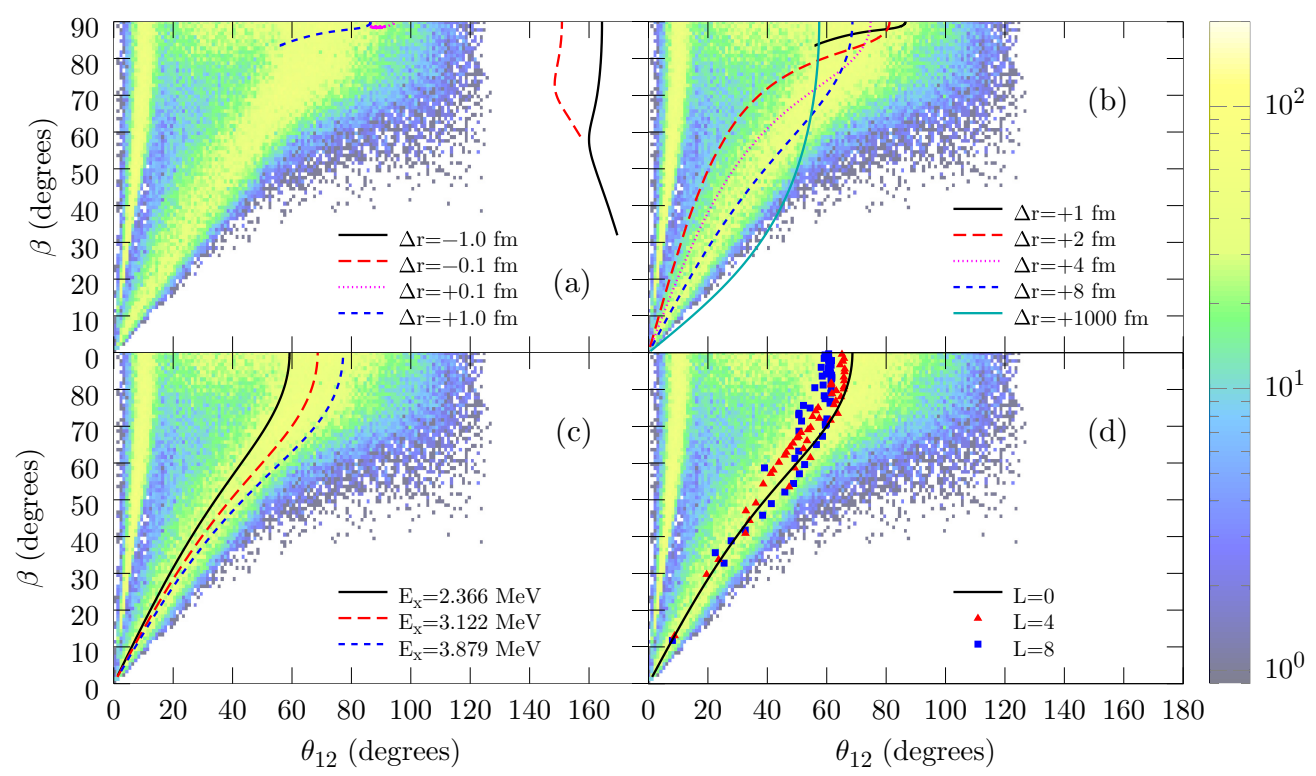

FIG. 6. Summary of model calculations and their comparison with data for the correlation between $\beta$ and $\theta_{12}$. (a) Curves for breakup distances near the distance of closest approach, where a negative value of $\Delta r$ indicates breakup prior to $R_{0}$ and a positive value after. (b) Curves for fixed breakup distances $\Delta r$ after the distance of closest approach. (c) Illustration of the sensitivity to the excitation energy (initial relative energy) of the fragments, covering the range of $E_{R}-\Gamma / 2$ to $E_{R}+\Gamma / 2$ for the $2^{+}$resonance. (d) Illustration of the sensitivity to projectile-target angular momenta, represented by the points. The calculations for (c) and (d) were made with fixed $\Delta r=8 \mathrm{fm}$. See text for further discussion.

symmetry and the calculations lie along a single line. The disintegration prior to $R_{0}$ leads to significantly larger angular separations $\theta_{12}$ than disintegration after $R_{0}$. The present experiment cannot detect this component as the detectors are arranged in a configuration allowing for a maximum $\theta_{12}=132^{\circ}$. This work indicates that future experiments with nickel-region targets must cover angles in both the forward and backward hemispheres to probe breakup prior to the distance of closest approach, which is relevant to fusion suppression.

Near $R_{0},{ }^{8} \mathrm{Be}$ moves slowly and the majority of the energy is stored in the potential between the fragments and the target. This energy is released in the (essentially) independent interaction of the fragments with the target following disintegration, resulting in $\alpha$ fragments with more similar energies if disintegration occurs near the turning point $R_{0}$. As a result we see a high propensity for events to reconstruct near $\beta=90^{\circ}$, with no events reconstructing to $\beta<80^{\circ}$ for $\Delta r=+1 \mathrm{fm}$. In part this is because of incomplete fusion of one fragment with the target.

The rapid changes near the turning point (e.g., from $\Delta=-0.1$ to $+0.1 \mathrm{fm})$ are in part a consequence of the simulations - a small effective $Q$ value is added to the radial motion to attain the correct total energy $E_{1}+E_{2}$ (see the Appendix). Prior to the distance of closest approach, this energy change causes the center of mass of the fragments to be accelerated towards the target; after $R_{0}$ it is accelerated away from the target. In reality the sensitivity near the turning point may be smaller than is suggested by Fig. 6(a).

\section{B. Outgoing breakup}

Because breakup near the distance of closest approach leads to $\alpha$ fragments with similar energies and $\beta \approx 90^{\circ}$, we next consider breakup on the outgoing trajectory, where the ${ }^{8} \mathrm{Be}$ is receding from the target. We make simulations for a series of disintegration radii after the distance of closest approach: $\Delta r=+1.0,+2.0,+4.0$, and $+8.0 \mathrm{fm}$. A further calculation was made for $\Delta r=+1000.0 \mathrm{fm}$ which is essentially the asymptotic limit. The results are shown in Fig. 6(b). As the disintegration radius increases the curve in $\beta$ and $\theta_{12}$ transitions from the near target result (black line) concentrated near $\beta=90^{\circ}$, towards the asymptotic limit (turquoise solid line). Comparing the simulations to the band of events seen in the experimental data suggests that much of the breakup observed with the present experimental configuration occurs several femtometers after the distance of closest approach.

For each event the initial orientation of the $\alpha$ fragments with respect to the target is randomly chosen. The projectile-target trajectory axial symmetry means that these events all fall on a single line. However, the events are not uniformly distributed along this line: There is a tendency for events to cluster towards $\beta=90^{\circ}$ corresponding to $E_{1}$ and $E_{2}$ being approximately equal. This may, in part, explain the increased intensity near $\beta=90^{\circ}$ found in the data. The detail of this is beyond the scope of the present discussion, and will be addressed in a future work.

Some of the results from Fig. 6(b) are also shown plotted for the relative energy $E_{\text {rel }}$ and $\left|E_{1}-E_{2}\right|$ in Fig. 7. The interaction of the fragments with the target generate a range of relative energies for each breakup distance, with the width of the range increasing as the breakup occurs closer to the target. This again illustrates how the resulting $E_{\text {rel }}$ depends sensitively on the initial conditions at the point of breakup, a fact previously noted in related classical simulations post-acceleration in Coulomb breakup [30]. 


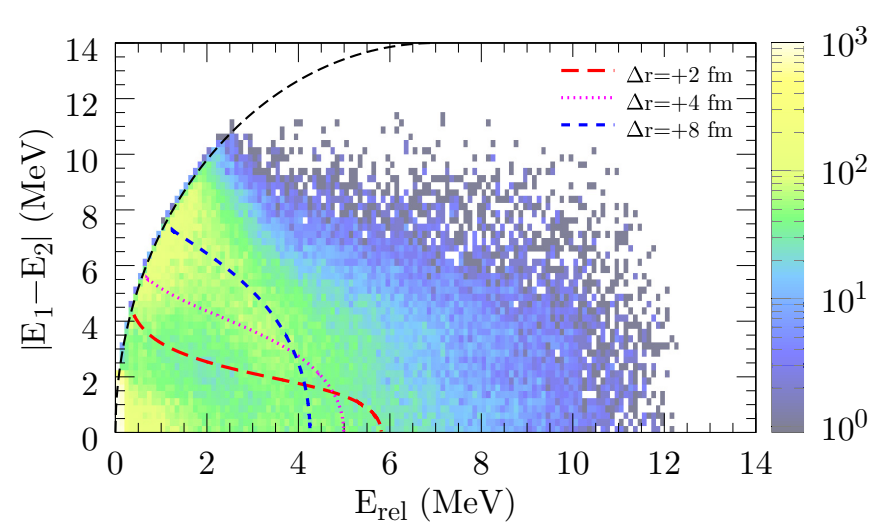

FIG. 7. The distribution of events according to their relative energy $E_{\text {rel }}$ and difference of energies $\left|E_{1}-E_{2}\right|$. A fixed excitation energy of $3.122 \mathrm{MeV}$ was used and projectile-target angular momentum $L=0$. The dashed black arc again shows the maximal allowed $\left|E_{1}-E_{2}\right|$.

\section{Initial excitation}

A further relevant variable is the initial excitation energy of ${ }^{8} \mathrm{Be}$, which we have thus far assumed to be equal to the energy of the $2^{+}$state at $E_{R}=3.122 \mathrm{MeV}$. This state has a finite width, with $\Gamma=1.513 \mathrm{MeV}$, so a distribution of excitation energies should result from its population. Calculations were made for $\Delta r=+8 \mathrm{fm}$ for initial excitation energies equal to $E_{R}$ and $E_{R} \pm \Gamma / 2(3.122,2.366$, and $3.879 \mathrm{MeV})$ to gauge the variation associated with the anticipated energy spread. These results are shown in Fig. 6(c). The qualitative pattern is similar for each of the excitation energies, with smaller $E_{x}$ giving smaller $\theta_{12}$. Thus the width of any resonant state populated can be expected to broaden the $\beta$ vs $\theta_{12}$ correlation for a fixed breakup distance.

\section{Projectile scattering angle}

The simulations presented so far assumed central collisions, and the axial symmetry this imposes leads to all events lying on a single line. With nonzero projectile-target angular momenta, this axial symmetry is broken. In Fig. 6(d) we show the dependence on the projectile-target angular momentum (i.e., impact parameter). As a guide, pure Rutherford scattering for the angular momenta shown corresponds to laboratory frame scattering angles $180^{\circ}(\mathrm{L}=0 \hbar), 150^{\circ}(\mathrm{L}=4 \hbar)$, and $104^{\circ}(\mathrm{L}=8 \hbar)$. This approximately corresponds to the coverage of the detectors in the present configuration. The nonzero scattering angle trajectories give a distribution of events shown by the points. The result is a broadening of the distribution for a fixed breakup distance, but the qualitative pattern remains the same. The distribution of the points gives some indication of tendency to cluster towards $\beta=90^{\circ}$ (and therefore $E_{1} \approx E_{2}$ ). As noted earlier, the initial orientation of the $\alpha-\alpha$ vector was assumed to be isotropic. An anisotropic distribution of $\alpha$ particles with respect to the targetlike nucleus will alter how the events are clustered along the band.

\section{DISCUSSION}

For the present case of $\alpha-\alpha$ coincidences from collisions of ${ }^{7} \mathrm{Li}$ with ${ }^{58} \mathrm{Ni}$, the experimental data are consistent with proton pickup via ${ }^{8} \mathrm{Be}$. The mechanism was previously studied with ${ }^{40} \mathrm{Ca}\left({ }^{7} \mathrm{Li},{ }^{8} \mathrm{Be}_{g s}\right){ }^{39} \mathrm{~K}$ [31], where good agreement was found between finite-range distorted-wave Born approximation calculations and measured angular distributions. In the present work, the $\alpha-\alpha$ relative energy distribution clearly shows a peak associated with the ${ }^{8} \mathrm{Be}$ ground state. The prompt breakup component of the data is well explained by classical simulations that assume breakup via the ${ }^{8} \mathrm{Be} 2^{+}$ state. Other mechanisms, such as direct breakup into $\alpha+t$ followed by proton pickup by the triton, do not appear to be necessary.

Furthermore, study of the $\alpha-\alpha$ angular correlations ( $\beta$ vs $\left.\theta_{12}\right)$ allows, in principle, some separation between breakup on the incoming and outgoing trajectories: Breakup on the incoming trajectory leads to larger fragment opening angles $\theta_{12}$ than on the outgoing trajectory. This feature may be restricted to relatively light target nuclei-heavy targets such as ${ }^{208} \mathrm{~Pb}$ will tend to focus both fragments towards backward angles regardless of the breakup location because of the stronger fragment-target Coulomb interaction. Lighter targets therefore potentially offer a better chance to understand the breakup dynamics. However, the current measurement using a ${ }^{58} \mathrm{Ni}$ target, with coverage only the backward hemisphere, is found to be sensitive, primarily, to breakup when the ${ }^{8} \mathrm{Be}$ is receding from the target. Simulations suggest that the majority of breakup prior to the distance of closest approach would have been missed with this detector configuration.

While the ideas outlined in this paper are a start, further work is required before precise conclusions regarding the breakup locations can be drawn. In reality, a distribution of initial excitation energies (e.g., [22,24,32]), disintegration points, and projectile-target angular momenta should be sampled. All will tend to broaden the distribution of events in $\beta$ vs $\theta_{12}$ for a given disintegration distance $\Delta r$, but the present simulations indicate that the qualitative pattern found should remain. More complete simulations to understand the details of these effects are being developed.

A key component of future simulations will be proper treatment of the lifetime of broad resonance states such as the ${ }^{8} \mathrm{Be} 2^{+}$resonance. The width of this state is $\Gamma=1.513 \mathrm{MeV}$, implying a mean lifetime $\tau \approx 0.4 \mathrm{zs}$. Although short-lived, its lifetime is of the order of the projectile-target collision time scale. A delay in breakup of this order could be sufficient to allow the ${ }^{8} \mathrm{Be}$ to pass the turning point and begin to recede from the target before breaking into $\alpha$ particles. A related effect was observed in high energy Coulomb dissociation of ${ }^{7} \mathrm{Li}$, which was studied extensively [30,33-38]. There it is found that the breakup in the astrophysically interesting low excitation range does not occur instantaneously. The lifetime of the state produced depends sensitively on its excitation and angular momentum, with the barrier to be tunneled through increasing at lower excitation and higher angular momenta $[39,40]$. Similarly, in above-barrier breakup of ${ }^{6} \mathrm{Li}$ into $\alpha+d$ [41], it was concluded that the breakup of the low-lying continuum occurs at large internuclear distances. The prompt breakup of the ${ }^{8} \mathrm{Be} 2^{+}$state itself was studied following emission from hot compound nuclei produced in high energy collisions of ${ }^{114} \mathrm{Cd}$ and ${ }^{92} \mathrm{Mo}$ [42]. There, even including the lifetime of 
the state, it appeared that breakup of ${ }^{8} \mathrm{Be}$ was inhibited close to the emitting compound nucleus.

This lifetime effect of the ${ }^{8} \mathrm{Be} 2^{+}$resonance is consistent with the present simulations, and may explain why much of the breakup appears to occur several femtometers after the distance of closest approach. Other projectiles and breakup channels may be similarly affected-for example, those proceeding via ${ }^{5} \mathrm{Li}$. This would have very important consequences for fusion reactions at above-barrier energies. If the $\alpha$ particles remain spatially localized long enough for them both to move inside the fusion barrier, the capacity for breakup to hinder fusion may be significantly reduced.

\section{SUMMARY}

In summary, we have presented new results for $\alpha-\alpha$ coincidences following proton pickup by ${ }^{7} \mathrm{Li}$ on a ${ }^{58} \mathrm{Ni}$ target at energies below the fusion barrier. The relative energy distributions of the $\alpha$ particles show a long-lived component associated with the $0.092-\mathrm{MeV}$ ground state of ${ }^{8} \mathrm{Be}$ and a short-lived component arising from disintegration near the target nucleus. Previously it had been assumed that for prompt breakup, half of the events originated from breakup on the incoming trajectory prior to reaching the turning point $R_{0}$, and half after. Fusion suppression predictions were made assuming those breaking up on the inbound trajectory were able to suppress fusion.

The present work develops this interpretation further, showing that the correlation between the fragment opening angle $\theta_{12}$ and the orientation of their relative velocity $\beta$ is sensitive to the proximity to the target when breakup occurs. This offers a new approach to study experimentally the prompt breakup component. Comparison with classical dynamical model simulations [26] indicate that the present experiment with a relatively light ${ }^{58} \mathrm{Ni}$ target, and where both $\alpha$ particles are detected at backward angles, is sensitive to breakup on the outgoing trajectory. To probe incoming trajectory breakup, future experiments must have a larger angular coverage, able to observe coincidence events where the fragments have large angular separations. A more complete treatment within the classical dynamical model is in preparation, incorporating an improved description of the transfer product initial excitation and estimates of the corresponding lifetime.

\section{ACKNOWLEDGMENTS}

Helpful discussions with A. Diaz-Torres are gratefully acknowledged. We thank M. Evers, L. R. Gasques, P. R. S. Gomes, R. Linares, and A. Wakhle for their assistance in running the experiment. Support from Australian Research Council Grants No. FL110100098, No. DP130101569, No. DE140100784, and No. DP14101337 is acknowledged. Support for accelerator operations through the NCRIS program is acknowledged.

\section{APPENDIX: CLASSICAL DYNAMICAL SIMULATIONS}

The classical dynamical simulations use a modified version of the PLATYPUS code [16,26], in which transfer is treated as a quasibreakup event. That is, the incident particle is ${ }^{8} \mathrm{Be}$, the energy of which is chosen to match the distance of closest approach of the ${ }^{7} \mathrm{Li}$ projectile assuming a Rutherford trajectory. Given a ${ }^{7} \mathrm{Li}$ beam energy of $13.1 \mathrm{MeV}$, the distance of closest approach $R_{0}=10.3 \mathrm{fm}$. Attaining an equivalent distance of closest approach with ${ }^{8} \mathrm{Be}$ requires a laboratory energy of 17.1 MeV. The nuclear interaction potentials involving the targetlike nucleus ${ }^{57} \mathrm{Co}$ and light nuclei $\left({ }^{8} \mathrm{Be}\right.$ and $\left.\alpha\right)$ are taken to be of Woods-Saxon, with geometry parameters fit to reproduce the Sao Paulo potential [17]. The $\alpha$ - $\alpha$ potential was a Woods-Saxon fit to the low-energy $\alpha$ - $\alpha$ scattering potential of Ref. [43]. However, the present simulations made at energies below the projectile-target barrier energy are not sensitive to the details of the nuclear part of the potential.

The $\alpha-\alpha$ potential also determines the initial separation of the fragments. To clearly define the starting conditions, we set the initial radial separation of the $\alpha$ particles to the radius of their mutual barrier radius $R_{B} \approx 6 \mathrm{fm}$ and insist they move radially outwards. This radius is determined by the $\alpha-\alpha$ potential used, and is similar to the value of $5.81 \mathrm{fm}$ used in Ref. [42]. Again, the present results are insensitive to these details.

Because of its intrinsic $\alpha$-cluster structure, ${ }^{8} \mathrm{Be}$ has a large binding energy per nucleon, and ${ }^{7} \mathrm{Li}$ proton pickup reactions typically have large and positive $Q$ values as a result. For the case of ${ }^{7} \mathrm{Li}+{ }^{58} \mathrm{Ni}$, the ground-state $Q$ value is $9.174 \mathrm{MeV}$. The optimal $Q$ value is estimated to be $3.34 \mathrm{MeV}$. To obtain the correct total energy of the fragments $E_{1}+E_{2}$ following disintegration for the chosen $Q=6.2-\mathrm{MeV}$ band in the data, an effective $Q$ value $Q_{\text {eff }}=4 \mathrm{MeV}$ must be included. Note that this effective $Q$ value differs from what one might expect from the ground-state $Q$ value and target excitation, because of the ${ }^{8}$ Be pseudoprojectile. As such, $Q_{\text {eff }}$ was chosen to produce the measured total energy of the fragments $E_{1}+E_{2}$. PLATYPUS conserves angular momentum at the point of breakup, so the excitation energy and effective $Q$ value are added to (or subtracted from) the projectile-target radial energy. The change in the radial energy of the projectile and target relative motion is then the difference between the effective $Q$ value and the excitation energy. For the present case this change is small.
[1] M. Dasgupta et al., Fusion Versus Breakup: Observation of Large Fusion Suppression for ${ }^{9} \mathrm{Be}+{ }^{208} \mathrm{~Pb}$, Phys. Rev. Lett. 82, 1395 (1999).

[2] C. Signorini et al., Does break-up affect ${ }^{9} \mathrm{Be}+{ }^{209} \mathrm{Bi}$ fusion at the barrier? Eur. J. Phys. A 5, 7 (1999).

[3] M. Dasgupta et al., Fusion and breakup in the reactions of ${ }^{6} \mathrm{Li}$ and ${ }^{7} \mathrm{Li}$ nuclei with ${ }^{209} \mathrm{Bi}$, Phys. Rev C 66, 041602(R) (2002).
[4] Y. W. Wu et al., Near barrier fusion excitation function of ${ }^{6} \mathrm{Li}+$ ${ }^{208} \mathrm{~Pb}$, Phys. Rev C 68, 044605 (2003).

[5] M. Dasgupta et al., Effect of breakup on the fusion of ${ }^{6} \mathrm{Li}$, ${ }^{7} \mathrm{Li}$, and ${ }^{9} \mathrm{Be}$ with heavy nuclei, Phys. Rev. C 70, 024606 (2004).

[6] M. Dasgupta, D. J. Hinde, S. L. Sheehy, and B. Bouriquet, Suppression of fusion by breakup: Resolving the discrepancy 
between the reactions of ${ }^{9} \mathrm{Be}$ with ${ }^{208} \mathrm{~Pb}$ and ${ }^{209} \mathrm{Bi}$, Phys. Rev C 81, 024608 (2010).

[7] R. Ost, K. Bethge, H. Gemmeke, L. Lassen, and D. Scholz, Three-particle correlations from ${ }^{208} \mathrm{~Pb}+{ }^{6} \mathrm{Li}$, Z. Phys. A 266, 369 (1974).

[8] D. J. Hinde, M. Dasgupta, B. R. Fulton, C. R. Morton, R. J. Wooliscroft, A. C. Berriman, and K. Hagino, Fusion Suppression and Sub-Barrier Breakup of Weakly Bound Nuclei, Phys. Rev. Lett. 89, 272701 (2002).

[9] C. Signorini et al., Exclusive breakup of ${ }^{6} \mathrm{Li}$ by ${ }^{208} \mathrm{~Pb}$ at Coulomb barrier energies, Phys. Rev. C 67, 044607 (2003).

[10] A. Shrivastava, A. Navin, N. Keeley, K. Mahata, K. Ramachandran, V. Nanal, V. V. Parkar, A. Chatterjee and S. Kailas, Evidence for transfer followed by breakup in ${ }^{7} \mathrm{Li}+{ }^{65} \mathrm{Cu}$, Phys. Lett. B 633, 463 (2006).

[11] R. Rafiei, R. du Rietz, D. H. Luong, D. J. Hinde, M. Dasgupta, M. Evers, and A. Diaz-Torres, Mechanisms and systematics of breakup in reactions of ${ }^{9} \mathrm{Be}$ at near-barrier energies, Phys. Rev. C 81, 024601 (2010).

[12] D. H. Luong, M. Dasgupta, D. J. Hinde, R. du Rietz, R. Rafiei, C. J. Lin, M. Evers, and A. Diaz-Torres, Insights into the mechanisms and time-scales of breakup of ${ }^{6,7} \mathrm{Li}$, Phys. Lett. B 695, 105 (2011).

[13] D. H. Luong, M. Dasgupta, D. J. Hinde, R. du Rietz, R. Rafiei, C. J. Lin, M. Evers, and A. Diaz-Torres, Predominance of transfer in triggering breakup in sub-barrier reactions of ${ }^{6,7} \mathrm{Li}$ with ${ }^{144} \mathrm{Sm}$, ${ }^{207,208} \mathrm{~Pb}$, and ${ }^{209} \mathrm{Bi}$, Phys. Rev. C 88, 034609 (2013).

[14] S. Santra, V. V. Parkar, K. Ramachandran, U. K. Pal, A. Shivastava, B. J. Roy, B. K. Nayak, A. Chatterjee, R. K. Choudhury, and S. Kailas, Resonant breakup of ${ }^{6} \mathrm{Li}$ by ${ }^{209} \mathrm{Bi}$, Phys. Lett. B 677, 139 (2009).

[15] D. Martinez Heimann et al., Differential and total cross sections of noncapture breakup reactions in the ${ }^{6} \mathrm{Li}+{ }^{144} \mathrm{Sm}$ system, Phys. Rev. C 89, 014615 (2014).

[16] A. Diaz-Torres, D. J. Hinde, J. A. Tostevin, M. Dasgupta, and L. R. Gasques, Relating Breakup and Incomplete Fusion of Weakly Bound Nuclei through a Classical Trajectory Model with Stochastic Breakup, Phys. Rev. Lett. 98, 152701 (2007).

[17] L. C. Chamon, D. Pereira, M. S. Hussein, M. A. Cândido Ribeiro, and D. Galetti, Nonlocal Description of the NucleusNucleus Interaction, Phys. Rev. Lett. 79, 5218 (1997).

[18] D. H. Luong, K. J. Cook, E. Williams, M. Dasgupta, D. J. Hinde, R. du Rietz, R. Rafiei, and M. Evers, Break-up array for light nuclei: A new tool for exploring nuclear reactions of relevance to the cosmological ${ }^{7} \mathrm{Li}$ problem, in Proceedings of "XII International Symposium on Nuclei in the Cosmos," PoS (NICXII), 185 (2012).

[19] A. G. Blair and D. D. Armstrong, $\left(t, \mathrm{He}^{4}\right)$ Reaction on the even Ni isotopes, Phys. Rev. 151, 930 (1966).

[20] A. Marinov et al., Proton-hole states in ${ }^{57} \mathrm{Co}$ studied with the ${ }^{58} \mathrm{Ni}\left(d,{ }^{3} \mathrm{He}\right){ }^{57} \mathrm{Co}$ reaction at $78 \mathrm{MeV}$, Nucl. Phys. A 438, 429 (1985).

[21] M. R. Bhat, Nuclear data sheets for $A=57$, Nucl. Data Sheets 85, 415 (1998).

[22] A. M. Lane and R. G. Thomas, $R$-matrix theory of nuclear reactions, Rev. Mod. Phys. 30, 257 (1958).

[23] F. C. Barker and P. B. Treacy, Nuclear levels near thresholds, Nucl. Phys. 38, 33 (1962).
[24] F. C. Barker, Consistent description of unbound states observed in scattering and reactions, Aust. Jour. Phys. 41, 743 (1988).

[25] D. R. Tilley, J. H. Kelley, J. L. Godwin, D. J. Millener, J. Purcell, C. G. Sheu, and H. R. Weller, Energy levels of light nuclei $A=8,9,10$, Nucl. Phys. A 745, 155 (2004).

[26] A. Diaz-Torres, PLATYPUS: A code for reaction dynamics of weakly-bound nuclei at near-barrier energies within a classical dynamical model, Comp. Phys. Comm. 182, 1100 (2011).

[27] K. Hagino, M. Dasgupta, and D. J. Hinde, Fusion and breakup in the reactions of ${ }^{6,7} \mathrm{Li}$ and ${ }^{9} \mathrm{Be}$, Nucl. Phys. A 738, 475 (2004).

[28] L. Corradi et al., Near-barrier transfer and fusion of the systems ${ }^{33} \mathrm{~S}+{ }^{90,91,92} \mathrm{Zr}$, Z. Phys. A 335, 55 (1990).

[29] S. Saha, Y. K. Agarwal, and C. V. K. Baba, One- and two-proton transfer reactions in ${ }^{32} \mathrm{~S}+{ }^{64} \mathrm{Ni}$ and ${ }^{28} \mathrm{Si}+{ }^{68} \mathrm{Zn}$ at near-barrier energies, Phys. Rev. C 49, 2578 (1994).

[30] S. B. Gazes, J. E. Mason, R. B. Roberts, and S. G. Teichmann, Direct Processes in 54-MeV ${ }^{7} \mathrm{Li}$ Breakup Reactions on ${ }^{12} \mathrm{C}$ and ${ }^{197} \mathrm{Au}$ Targets, and the Extraction of Astrophysical Cross Sections, Phys. Rev. Lett. 68, 150 (1992).

[31] J. R. Hurd, D. R. James, J. J. Bevelacqua, and L. R. Medsker, $\left({ }^{7} \mathrm{Li},{ }^{8} \mathrm{Be}\right)$ reaction on ${ }^{40} \mathrm{Ca}$, Phys. Rev. C 20, 1208 (1979).

[32] R. J. Charity, L. G. Sobotka, J. Cibor, K. Hagel, M. Murray, J. B. Natowitz, R. Wada, Y. El Masri, D. Fabris, G. Nebbia, G. Viesti, M. Cinausero, E. Fioretto, G. Prete, A. Wagner, and $\mathrm{H}$. $\mathrm{Xu}$, Emission of unstable clusters from hot $\mathrm{Yb}$ compound nuclei, Phys. Rev C 63, 024611 (2001).

[33] H. Utsunomiya et al., Strongly-suppressed post-Coulomb acceleration in non-resonant breakup of ${ }^{7} \mathrm{Li}$, Phys. Lett. B 416, 43 (1998).

[34] H. Utsunomiya et al., Astrophysical implications of nonresonant break-up of ${ }^{7} \mathrm{Li}$, J. Phys. G 24, 1637 (1998).

[35] C. A. Bertulani, The photodissociation of ${ }^{8} \mathrm{~B}$ and the solar neutrino problem, Nucl. Phys. A 587, 318 (1995).

[36] Y. Tokimoto, H. Utsunomiya, T. Yamagata, M. Ohta, Y.-W. Lui, R. P. Schmitt, S. Typel, Y. Aoki, K. Ieki, and K. Katori, Coulomb breakup of ${ }^{7} \mathrm{Li}$ for nuclear astrophysics, Phys. Rev. C 63, 035801 (2001).

[37] J. E. Mason, S. B. Gazes, R. B. Roberts, and S. G. Teichmann, Coulomb and nuclear effects in direct breakup of $54-\mathrm{MeV}^{7} \mathrm{Li}+$ ${ }^{12} \mathrm{C},{ }^{197} \mathrm{Au}$, Phys. Rev. C 45, 2870 (1992).

[38] C. Baur, C. A. Bertulani, and H. Rebel, Coulomb dissociation as a source of information on radiative capture processes of astrophysical interest, Nucl. Phys. A 458, 188 (1986).

[39] D. D. Clayton, Principles of Stellar Evolution and Nucleosynthesis (University of Chicago Press, Chicago, 1983).

[40] C. E. Rolfs and W. S Rodney, Cauldrons in the Cosmos (University of Chicago Press, Chicago, 1988).

[41] F. A. Souza et al., Projectile breakup dynamics for ${ }^{6} \mathrm{Li}+{ }^{59} \mathrm{Co}$ : Kinematical analysis of $\alpha-d$ coincidences, Eur. Phys. J. A 44, 181 (2010)

[42] A. B. McIntosh, S. Hudan, C. J. Metelko, R. T. de Souza, R. J. Charity, L. G. Sobotka, W. G. Lynch, and M. B. Tsang, Tidal Effects and the Proximity Decay of Nuclei, Phys. Rev. Lett. 99, 132701 (2007).

[43] B. Buck, H. Fridrich, and C. Wheatley, Local potential models for the scattering of complex nuclei, Nucl. Phys. A 275, 246 (1977). 